\title{
Trial designs for the development of new drugs
}

\author{
Cecilia Orbegoso $^{1}$, Eleonora Ghisoni ${ }^{2}$, Susana Banerjee ${ }^{3}$
}

\section{Introduction}

The development of new drugs and therapeutic strategies have transformed over recent years largely due to the increased knowledge of tumor biology and molecular characterization. In the past, the 'one-size-fits all' approach of traditional trials in which interventions are tested in a single cancer at a particular point in the cancer journey (eg. first line, relapse) has led to the licensing of anti-cancer agents leading to the current standard of care for many cancers. However, this approach can be expensive and for many trials, in particular for rarer cancers, takes many years to deliver. More recently, there has been a need to refine clinical trial design at a time where high-throughput technologies allow researchers and clinicians to better understand tumor biology and access molecular profiling. The expectation is that more tailored clinical trials are more likely to identify practice-changing treatments in selected patient groups. Clinical trial designs have evolved to incorporate the rapidly changing wealth of molecular information. Here, we summarize the rationale, potential advantages and limitations of some examples of new clinical trial designs from a clinician's point of view. We recommend and refer to several recent reviews to gain better insight into statistical considerations [1-3].

\section{Master Protocols}

Master Protocols is a term that refers to a framework in which multiple parallel drugs studies are operated under one protocol, each parallel study consisting of different biomarker-treatment combinations. The key requirement for the successful implementation of such a protocol is the cross collaboration between and within the clinical research and scientific community, pharmaceutical industry and regulatory bodies.

Master Protocols use common screening platform evaluating multiple markers and have the ability to add and remove cohorts in a pre-planned, rapid manner. In this way, the burden, time and bureaucracy of developing separate protocols are minimized and as a consequence, clinical development is expedited. Importantly, the information obtained from each cohort and individual patient can be interrogated within the protocol to develop future options. Statistical considerations for this type of protocol are often more complex than traditional trial designs. The Master Protocol can permit different study objectives for each cohort, either discovery-based or confirmatory objectives and in some cases sequential objectives using a multi-phase design within a cohort [1]. Teamwork across disciplines in the design and conduct of Master Protocols is essential. Common protocol elements such as schedule of assessments, endpoint definitions and coordinated data collection are important factors for the success of such a trial. The number of patients required to participate in a trial is important and can be limiting for some study concepts that involve rare cancers/molecular subgroups. A consideration in the era of precision medicine is that trials should be designed to identify signals of activity and aim for the identification of large and clinically meaningful differences within small molecularly enriched groups and therefore maximise the information derived from potentially fewer patients [1]. Examples of Master Protocols are basket and umbrella trials.

\section{Basket trials}

In basket trials, patient eligibility is usually defined by the presence of a particular molecular alteration or tumor biomarker and often includes multiple tumor types [2-4]. These trials hypothesize that molecular characterization predicts response to a matched treatment. They do not necessarily have a control arm due to differences in standard therapy across tumor types $[1,2]$. Basket trials provide access to molecular-based targeted therapies which is particularly attractive for the study of rarer cancers [5].

${ }^{1}$ Gynaecology Unit, The Royal Marsden NHS Foundation Trust, London, UK.

${ }^{2}$ Division of Medical Oncology, Candiolo Cancer Institute-FPO-IRCCS, Candiolo (To), Italy.

${ }^{3}$ Gynaecology Unit, The Royal Marsden NHS Foundation Trust and Division of Clinical Studies, The Institute of Cancer Research, London, UK.

Correspondence to:

Susana Banerjee,

Gynaecology Unit, The Royal Marsden NHS Foundation Trust and Division of Clinical Studies, The Institute of Cancer Research, 203 Fulham Road, London, SW3 6JJ, UK.

Phone: +44 2078118579 - Fax: +44 2078118103

E-mail: Susana.Banerjee@rmh.nhs.uk

CANCER BREAKING NEWS 2017;5(3):41-44

DOI: $10.19156 / c b n .2017 .0059$ 
Cohorts are often small and designed in a single-stage or two-stage manner. An important aspect within these trials is often the validation of a companion diagnostic test [1].The design and interpretation can be more challenging given the multiple diseases and expectations of response rates for each condition. Moreover, the inclusion of rare tumors and molecular alterations may lead to high screening failure rates. However, these studies provide valuable initial information on the proof of concept to identify if a drug harbours clinical activity in a selected group of patients [6].The assumption that molecular characterization may be superior or equivalent to histological tumor typing needs to be considered. A particular mutation may be the molecular driver in one cancer type but not in another. The situation is complex and likely to differ across malignancies. An example of a basket trial is the Vemurafenib in BRAF V600 MutationPositive Cancers (NCT01524978). Vemurafenib (Roche) is associated with high response rates in BRAF V600E mutated melanoma and has also shown some activity in BRAF V600E mutated ovarian cancer, cholangiocarcinoma and anaplastic thyroid cancer with responses lasting for more than 12 months. In comparison, responses in BRAF V600E mutated colon cancer are limited [7, 8]. Other trials including pembrolizumab (Merck) have shown efficacy across tumours with mismatch repair deficiency. The Keynote 164 (NCT02628067) and Keynote 158 (NCT02460198) trials included patients with MSI-H colon, endometrial, small intestinal, cholangiocarcinoma, gastric, and pancreatic cancer. The objective response rates was $26 \%$ for the colon cancer cohort and $43 \%$ for the non colon cancer cohort [9]. The EORTC CREATE phase II trial (NCT01524926) evaluates crizotinib (Pfizer) activity in six different tumor types harboring alterations in ALK and/or MET pathways [10].The phase II NCI IMPACT trial (NCT01827384) is based on molecular profiling assignment of cancer therapy in patients with advanced solid tumors. This trial assigns patients to 1 out of 4 regimens corresponding to one of the mutations/amplifications with the opportunity of crossover upon progression to the second molecularly profiled regimen. Treatment arms include: velaparib with temozolomide, WEE1 inhibitor AZD1775 with carboplatin, everolimus, and trametinib [11].

\section{Umbrella trials}

The key characteristic of this design is that patients with a specific tumor type are screened and assigned to subtrials of different treatments, often based on the molecular profile and involving targeted therapies. The identification of an actionable biomarker may occur at the time of analysis after recruitment completion [1]. Umbrella trials permit the evaluation of new drugs in a specific group of patients with the same tumor type and thus limits the issue of biological differences leading to differences in activity across tumor types. This allows for better interpretation of the efficacy and mechanism of action of the selected drug in a specific population. Furthermore, these trial designs could allow for the predictive or prognostic value of a specific marker to be explored when a control arm (eg. without targeted therapy) is included [1]. The modular structure of the umbrella trial is complex-various arms can be moved in and out of the study as new drugs become available or due to toxicity concerns. Patient selection based on histology/molecular profile is an advantage, however, it can also be limiting in particular for rare diseases where accrual within the different cohorts can be a challenge thus potentially slowing down the progression of the trial- time in which the standard of care might have changed $[1,12]$. The OCTOPUS trial (EudraCT 2014-005221-12) is an umbrella phase II framework for testing whether the addition of novel targeted agents to weekly paclitaxel improves efficacy in platinum-resistant ovarian cancer; the first drug to be tested in combination with weekly paclitaxel is vistusertib a dual mTORC1/2 inhibitor [13]. The LUNG-MAP trial (NCT02154490) [14] represents another example of umbrella trials; four randomized phase II trials of targeted therapy versus standard of care are conducted in parallel within mutationenriched cohorts. Patients are allocated to different drugbiomarker combination arms. Drugs include checkpoint inhibitors (durvalumab, nivolumab, ipilimumab), targeted therapies, including tyrosine-kinase inhibitors, cyclindependent kinases inhibitors, amongst others (erlotinib, FDFR inhibitor AZD4547, palbociclib, taselisib, and rilotumumab) and docetaxel. Another example is the NSGO-OV-UMB1; ENGOT-OV30 / NSGO trial which is due to open shortly. This phase II umbrella trial involves patients with relapsed ovarian cancer (NCT03267589). Each coordinating group within the ENGOT framework leads a specific treatment arm. The treatment arms therefore, can run concurrently across different countries. At present, the arms include the following treatment combinations MEDI9447 (CD73) + durvalumab; MEDI0562 (OX40) + durvalumab and MEDI0562 (OX40) + tremelimumab.

\section{Adaptive clinical trials}

Adaptive clinical trials [15] are dynamically evolving trials designed with the aim of being flexible, efficient (patient numbers and cost) and fast. A key characteristic of the design is the equal ratio recruitment amongst the different arms during the initial phase, followed by an adaptive phase based on efficacy data generated from the different cohorts. During the adaptive phase, trial design is modified based on accumulating data; the recruitment 
ratios may change and arms could be dropped or added. One of the advantages of the design for trials of targeted therapy relies on its plasticity-molecular based profiling design for a flexible biomarker strategy independent of treatment assignment and dependent on emerging evidence of the sensitivity of the treatments under evaluation. An example of a trial with an adaptive design is the BATTLE study (NCT00409968, NCT00411671, NCT00411632, NCT00410059, NCT00410189) [16], a prospective, biopsymandated, biomarker-based, adaptively randomized study, in which following an initial equal randomization period, chemo-refractory patients with non-small cell lung cancer (NSCLC) were adaptively randomized to four different arms (erlotinib, vandetanib, erlotinib plus bexarotene and sorafenib) based on relevant molecular biomarkers analyzed in fresh core needle biopsy specimens. Others include BATTLE-2 (NCT01248247) (four treatment regimens in NSCLC-erlotinib, sorafenib, erlotinib + MK2206, and MK2206 + AZD6244). The I-SPY 2 trial (NCT01042379) is an adaptive phase 2 trial of neoadjuvant therapy for highrisk stage II/III breast cancer which evaluated multiple new agents added to standard chemotherapy to assess pathological complete response [17]. Neratinib added to standard therapy was identified as warranting further investigation having shown promising rates of pathological complete response [17].

\section{Other innovative trial designs}

Finally, window-of-opportunity studies are designed to assess the administration of an investigational drug during a short period of time, most often prior surgery. The objective of this type of trials is to study in vivo the biological effects of the drug in treatment naïve patients. Pre- and post-treatment biopsies serve for translational research to improve our understanding of pharmacodynamic parameters and to identify biomarkers for better patient selection leading to the further translational-based clinical trials $[15,18]$ with the aim of expediting drug development. Despite the advantages for translational research-based studies, the safety concerns of repeated biopsies, logistical issues for some cancer centres and the potential delay in starting standard therapy are limiting factors [18]. However, this approach is increasingly attractive and an example of such a trial is the POLEN study (NCT02506816) [19] which assesses the activity of preoperative olaparib (PARP inhibitor) in early stage endometrial cancer.

\section{Discussion}

As next-generation sequencing technologies continue to develop, molecular and genomic evaluation of patients will become part of routine clinical practice. These changes will allow clinicians to treat patients with tailored drugs according to molecular profiling which will hopefully improve patient outcomes. Because of this, clinical trials need to evolve, shifting from a more traditional paradigm, to a molecular-driven trial design. New trial designs offer the possibility of more flexible protocols that allow changes during the development of the study based on predefined criteria, with the possibility to expedite cancer drug development based on larger clinical benefits in selected patient populations. A challenge is incorporating agents developed from more than one pharmaceutical companies in the same trial. In addition to administrative logistics (eg. contracts, finance), the developmental pipeline and priorities may not always be aligned. However, it is clear that there has been substantial progress with industry and academic networks working together for the common goal of improving therapies for cancer patients.

\section{Conflicts of Interest}

The Authors declare there are no conflicts of interest in relation to this article. 


\section{References}

1. Renfo LA, Sargent DJ. Statistical controversial in clinical research: basket trials, umbrella trials, ando other master protocols: a review and examples. Ann Oncol 2017;28:34-43.

2. Redig AJ, Janne PA. Basket trials and the evolution of clinical trial design in an era of genomic medicine. J Clin Oncol 2015;33:975-7.

3. Catenacci DV. Next-generation clinical trials: Novel strategies to address the challenge of tumor molecular heterogeneity. Mol Oncol 2015;9(5):967-96.

4. Berry DA. The Brave New World of clinical cancer research: adaptive biomarker driven trials integrating clinical practice with clinical research. Mol Oncol 2015;9:951-9.

5. Billingham L, Malottki K, Steven N. Research methods to change clinical practice for patients with rare cancers. Lancet Oncol 2016;17:e70-e80.

6. Cunanan KM, Gonen M, Shen R et al. Basket trials in Oncology: A trade-off between complexity and efficiency. J Clin Oncol 2017;35:3271-3.

7. Hyman DM, Puzanov I, Subbiah V et al. Vemurafenib in multiple non-melanoma cancers with BRAF V600 mutations. N Engl J Med 2015;373:726-36.

8. Flaherty KT, Puzanov I, Kim KB et al. Inhibition of mutated, activated BRAF in metastatic melanoma. N Engl J Med 2010;363:809-19.

9. Diaz LA, Marabelle A, Delord JP et al. Pembrolizumab therapy for microsatellite instability high (MSI-H) colorectal cancer (CRC) and non-CRC. J Clin Oncol 2017;35:15 suppl, 3071-3071.

10. Schöffski P, Wozniak A, Casali PG et al. Activity and safety of crizotinib in patients with advanced clear cell sarcoma with MET alterations. European Organization for Research and Treatment of Cancer phase 2 trial 90101 "CREATE". Ann Oncol 2017 Sep 18.
11. Zhao Y, Polley EC, Li M-C et al. GeneMed: An informatics hub for the coordination of next-generation sequencing studies that support precision oncology clinical Trials. Cancer Informatics 2015;14(Suppl 2):45-55.

12. Saville BR, Berry SM. Efficiencies of platform clinical trials: a vision of the future. Clin Trials 2016;13:358-66.

13. Banerjee S, Lewsley LA, Clamp AR et al. OCTOPUS: A randomised, multi-centre phase II umbrella trial of weekly paclitaxel+/- novel agents in platinum-resistant ovarian cancer_-Vistusertib (AZD2014). J Clin Oncol 2017;35:15_ suppl, TPS5609-TPS5609.

14. Herbst RS, Gandar DR, Hirsch FR et al. Lung Master Protocol (Lung-MAP)-A biomarker-driven protocol for accelerating development of therapies for squamous cell lung cancer: SWOG S1400. Clin Cancer Res 2015;21(7): 1514-24.

15. Zardavas D, Piccart-Gebhart M. Clinical trials of precision medicine through molecular profiling: focus on breast cancer. Am Soc Clin Oncol Educ Book 2015:e183-90.

16. Kim ES, Herbst RS, Wistuba II et al. The BATTLE trial: personalizing therapy for lung cancer. Cancer Discover 2011;1:44-53.

17. Park JW, Liu M, Yee D et al. Adaptive randomization of neratinib in early breast Cancer. N Engl J Med 2016; 375(1):11-22.

18. Schmitz S, Duhoux F, Machiels JP. Window of opportunity studies: Do they fulfil our expectations? Cancer Treat Rev 2016;43:50-7.

19. Romero I, Rubio MJ, Serrano R et al. Preoperative olaparib in early-stage endometrial cancer: A phase 0, window of opportunity trial to evaluate the PARP inhibition effect, targeting cell cycle-related proteins (POLEN study). Ann Oncol 2016;27(suppl_6),903TiP. 